Published in final edited form as:

Curr Opin Rheumatol. 2009 January ; 21(1): 1-2. doi:10.1097/BOR.0b013e32831e4250.

\title{
Vasculitis: lessons learned
}

\author{
Philip Seo \\ Division of Rheumatology, Johns Hopkins University School of Medicine, The Johns Hopkins \\ Vasculitis Center, Baltimore, Maryland, USA
}

\begin{abstract}
The routine use of cyclophosphamide dramatically changed the prognosis associated with systemic vasculitis, transforming many of these previously fatal diseases into chronic conditions. This advance, however, has come at a significant cost. Even with standard-of-care therapies, patients with systemic vasculitis continue to accumulate chronic morbidity, either as a result of the disease or the medications used for treatment [1]. Biologic therapies represent an important advance - replacing a blunt instrument with a scalpel - and have opened the possibility of treating severe forms of vasculitis with something other than a cytotoxic agent.

It would, however, be premature to declare success. Early experience with biologic agents for the treatment of systemic vasculitis demonstrates that even under the best of circumstances, the reprieve is temporary. It seems fair to say that these agents do not change the fundamental characteristics of the underlying illness; they function more like levees against the storm waters, which work only as long as they remain in place. Moreover, the long-term consequences of many of these agents are not yet clear, and although many may seem both safe and effective after several years of use, the effects of several decades of use remain to be seen [2].
\end{abstract}

Although biologic therapies represent an important stepping stone, many fundamental challenges remain. The last several years have highlighted a few important themes.

\section{The importance of a multicenter, multidisciplinary approach}

It has become increasingly obvious that no single center is equipped to address fundamental questions regarding the primary systemic vasculitides. The advent of multicenter and multinational trials has been an important milestone in the study of many rare diseases, including vasculitis. It is important to note, however, that the majority of randomized clinical trials in vasculitis have addressed Wegener's granulomatosis and microscopic polyangiitis, which, given their prevalence, may be the low-hanging fruit. Future challenges lie in our ability to study less common forms of systemic vasculitis (such as rheumatoid vasculitis [3], primary angiitis of the central nervous system [4], and others) in an equally rigorous fashion.

At the same time, it has become equally obvious that rheumatologists alone are ill-equipped to treat patients with vasculitis [5]. As the immediate prognosis of patients with vasculitis continues to improve, the long-term consequences associated with these diseases become increasingly important. The impact of rheumatic disease on cardiovascular health is of particular interest [6], but in the future, it will be crucial to address the impact of vasculitis

Correspondence to Philip Seo, MD, MHS, 5501 Hopkins Bayview Circle, JHAAC Room 1B.1A, Baltimore, MD 21224, USA Tel: +1 410550 6813; fax: +1 866752 7405; seo@jhmi.edu.

Publisher's Disclaimer: This is a PDF file of an unedited manuscript that has been accepted for publication. As a service to our customers we are providing this early version of the manuscript. The manuscript will undergo copyediting, typesetting, and review of the resulting proof before it is published in its final citable form. Please note that during the production process errors may be discovered which could affect the content, and all legal disclaimers that apply to the journal pertain. 
(and its treatment) on sarcopenia, neuropathy, renal survival, and other forms of chronic morbidity that impact patients' quality of life.

Key to all of these advances is improvement in the metrics used to assess fundamental aspects of disease such as damage, quality of life, and patient-reported outcomes. International or multicenter collaboration is possible only if a common language is established that will allow patients to be assessed systematically and consistently across centers [7].

\section{The importance of phenotype}

There is an old adage about everyone being either a 'lumper' or a 'splitter'. Clinical trials generally require us to be 'lumpers', since patients with somewhat disparate phenotypes are assigned treatment strategies based on broad disease categories. Although the use of randomized clinical trials for the study of vasculitis is an important advance, it is also important to contemplate what we have lost. By homogenizing patients into broad disease categories, we may lose the ability to identify small subsets of patients for whom a given treatment may be successful. As we evolve toward personalized medicine [8], dissecting the clinical phenotype of each patient will become an increasingly important component of optimizing therapy and may yield important insights into diseases that we believe we already know well. For example, although Wegener's granulomatosis was first described in 1931 [9], only recently has it become apparent that venous thrombotic events may be an important manifestation of disease flare for patients with this diagnosis [10].

It also seems possible that in some cases, labels such as 'Wegener's granulomatosis' represent a spectrum of disorders in which disparate pathways lead to the same clinical phenotype. Molecular phenotyping, using advances in genomics and proteomics, may eventually become an important tool in the evaluation of patients with vasculitis and may be especially important in selecting treatment strategies, or identifying patients with disease variants now characterized as `atypical' or 'refractory'.

\section{The importance of surrogate markers}

The prevalence of diseases such as Wegener's granulomatosis and microscopic polyangiitis appears to have increased, at least in part due to improvements in diagnostic techniques [11]. Despite this, many patients will have signs and symptoms attributable to vasculitis for years before a clinical diagnosis is made [12]. The development of widely available, high-throughput diagnostic markers is crucial to shortening the interval between the initial disease manifestations and diagnosis. This in turn may lead to earlier treatment and thereby improve our ability to prevent the devastating consequences associated with these diseases.

Another important challenge is the lack of completely objective measures of disease states (i.e., remission, disease flare, and low disease activity or 'grumbling' disease). The development of surrogate markers of disease states would be an important step toward both facilitating early treatment and preventing overtreatment of patients with systemic vasculitis. Such surrogate markers need not be limited to serologic tests alone; advances in imaging modalities such as ultrasound, magnetic resonance imaging, and positron emission tomography, bring us closer to the time when these techniques may replace biopsy as the diagnostic modality of choice [13].

Despite improvements in vasculitis therapeutics, it has become increasingly apparent how far we still have to go. In the future, our perspective must broaden to include not only less common forms of systemic vasculitis, but also new challenges regarding early diagnosis and prevention of chronic morbidity. It feels like we are on the brink of a watershed in this field and perhaps the only safe conclusion is that the end is not yet in sight. 


\section{Acknowledgments}

Dr Seo is a Lowe Family Scholar in the Johns Hopkins University Center for Innovative Medicine.

The National Institutes of Health/National Institute of Arthritis and Musculoskeletal and Skin Diseases Grant Number K23 AR052820 (Johns Hopkins University School of Medicine).

\section{References}

1. Seo P, Min YI, Holbrook JT, et al. Damage caused by Wegener's granulomatosis and its treatment: prospective data from the Wegener's Granulomatosis Etanercept Trial (WGET). Arthritis Rheum 2005;52:2168-2178. [PubMed: 15986348]

2. Chung SA, Seo P. Advances in the use of biologic agents for the treatment of systemic vasculitis. Curr Opin Rheumatol. 2009 (in press).

3. Turesson C, Matteson EL. Vasculitis in rheumatoid arthritis. Curr Opin Rheumatol. 2009 (in press).

4. Hajj-Ali RA, Calabrese LH. Central nervous system vasculitis. Curr Opin Rheumatol. 2009 (in press).

5. Reinhold-Keller E, Beuge N, Latza U, et al. An interdisciplinary approach to the care of patients with Wegener's granulomatosis: long term outcome in 155 patients. Arthritis Rheum 2000;43:1021-1032. [PubMed: 10817555]

6. Kane GC, Keogh KA. Involvement of the heart by small and medium vessel vasculitis. Curr Opin Rheumatol. 2009 (in press).

7. Seo P, Luqmani RA, Flossmann O, et al. The future of damage assessment in vasculitis. J Rheumatol 2007;34:1357-1371. [PubMed: 17552062]

8. Tinetti ME, Fried T. The end of the disease era. Am J Med 2004;116:179-185. [PubMed: 14749162]

9. Klinger H. Borderline forms of periarteritis nodosa. Frankf Z Pathol 1931;43:455-480.

10. Tomasson G, Monach PA, Merkel PA. Thromboembolic disease in vasculitis. Curr Opin Rheumatol. 2009 (in press).

11. Al Maini M, Carette S. Diagnosis of Wegener's granulomatosis in the ANCA era. J Rheumatol 2006;33:1923-1924. [PubMed: 17014008]

12. Hoffman GS, Kerr GS, Leavitt RY, et al. Wegener's granulomatosis: an analysis of 158 patients. Ann Intern Med 1992;116:122-129.

13. Blockmans D, Bley T, Schmidt W. Imaging for large vessel vasculitis. Curr Opin Rheumatol. 2009 (in press). 